Most intriguingly, both groups demonstrate a relative expansion in $E m \times 2^{-/-}$mice of anterior cortical regions that normally express the lowest concentration of Emx2; Bishop et al. also demonstrate an absolute twofold expansion of anterior cortex (assessed using expression of an anterior cortical marker, Cad8) despite an overall one-third reduction of cortical surface area in these mice. Thus, cortical areas are differentially affected in $E m \times 2^{-/-}$mice, with posteromedial regions markedly reduced, intermediate cortex shifted, and anterior cortex expanded. The loss of Emx2 does not result in complete loss of any particular cortical area, but instead results in a change of scale of cortical areas relative to each other. Most importantly, these changes correspond to the normal Emx2 expression gradient (Fig. 1). Because changes in areal size in the $E m \times 2^{--}$mice correlate with areal position along the Emx2 gradient, it is likely that the Emx 2 gradient directly regulates areal size. A further implication of these findings is that cortical areas are seemingly able to compete for space, because the areas that are least dependent on Emx2 (anterior cortex) are able to expand at the expense of those that are most dependent (posterior cortex).

This model, suggesting that parcellation of cortical areas is regulated by homeodomain protein gradients, is supported by work on a second mouse mutant, which lacks the homeodomain protein Pax6. Cortical Pax6 expression is also graded, but in a direction that opposes Emx2 (high anterolateral, low posteromedial, Fig. 1). In a fashion similar to the Emx2 mutants, markers of anterior and lateral cortical regions that normally express high Pax6 levels (including motor and somatosensory cortex) are reduced but not completely lost in Pax6-deficient mice $^{9}$ (Fig. 1). Although study of the Pax6 mutant line was limited (in part because thalamic axons do not reach the cortex in these mice), these findings support a model in which the complementary Emx2 and Pax6 gradients provide positional information to the developing neocortex in much the same way that gradients of other homeodomain proteins, such as bicoid, impart positional information in other developmental contexts.

The next set of questions involves how these homeodomain proteins regulate the formation of specific cortical areas. Do they influence proliferation or some other aspect of area-specific programming? Mallamaci et al. have begun to address this issue by studying the tissue morphology and cell cycle kinetics of anterior, intermediate and posterior cortical progenitors in $E m \times 2$ knockout mice. Interestingly, they find that the proliferative layers are significantly thicker in these mice, but that the cell cycling times are indistinguishable from wild-type mice. This suggests that Emx2 does not regulate arealization by altering proliferation in a simple way, but may instead modulate other area-specific programs; additional experimental work will be needed to answer this question.

Several other outstanding questions remain unanswered. For instance, do Emx 2 and Pax6 act independently or in a combinatorial manner to impart positional information? An Emx2/Pax6 double knockout might be useful to answer this question. Also, how is the positional information encoded by these homeodomain protein gradients converted into the sharp areal boundaries of mature cortex? Mechanisms used to translate other homeodomain gradients (such as the bicoid gradient) into discrete subdivisions may provide insights into this problem. Another question concerns the upstream factors that establish the Emx2 and Pax6 gradients. Secreted molecules such as the Bmps, Wnts and Fgfs have been shown to act in gradients to initiate the production of transcription factors, and they may therefore be involved. Although the downstream transcriptional targets of Emx2 and Pax6 remain unknown, one potential target may be the ephrins, which show graded expression within somatosensory cortex and affect its topographic organization ${ }^{15}$. Finally, although the Emx2/Pax6 countergradient axis is clearly an important one, gradients along other axes must also be acting to subdivide cortex, because functional areas are not aligned solely along one axis. The identification of factors that act along other axes, and their interactions with the Emx2/Pax6 countergradient, will also be of great future interest.

1. Rakic, P. Science 241, 170-176 (1988).

2. O'Leary, D. D. Trends Neurosci. 12, 400-406 (1989).

3. Sharma, J., Angelucci, A. \& Sur, M. Nature 404, 841-847 (2000).

4. Barbe, M. F. \& Levitt, P. J. Neurosci. 11, 519-533 (1991).

5. Gitton, Y., Cohen-Tannoudji, M. \& Wassef, M. J. Neurosci. 19, 4889-4898 (1999).

6. Dehay, C., Giroud, P., Berland, M., Smart, I. \& Kennedy, H. Nature 366, 464-466 (1993).

7. Miyashita-Lin, E. M., Hevner, R., Wassarman, K. M., Martinez, S. \& Rubenstein, J. L. Science 285, 906-909 (1999).

8. Nakagawa, Y., Johnson, J. E. \& O’Leary, D. D. J. Neurosci. 19, 10877-10885 (1999).

9. Mallamaci, A., Muzio, L., Chan, C.-H., Parnvelas, J. \& Boncinelli, E. Nat. Neurosci. 3, 679-686 (2000).

10. Bishop, K. M., Goudreau, G. \& O’Leary, D. D. Science 288, 344-349 (2000)

11. Pellegrini, M., Mansouri, A., Simeone, A., Boncinelli, E. \& Gruss, P. Development 122, 3893-3898 (1996).

12. Brunelli, S. et al. Nat. Genet. 12, 94-96 (1996).

13. Simeone, A., Acampora, D., Gulisano, M., Stornaiuolo, A. \& Boncinelli, E. Nature 358, 687-690 (1992).

14. Tole, S., Goudreau, G., Assimacopoulos, S. \& Grove, E. A. J. Neurosci. 20, 2618-2625 (2000).

15. Vanderhaeghen, P. et al. Nat. Neurosci. 3, 358-365 (2000).

\title{
A new high for alternative splicing
}

Alternative splicing of RNA transcripts has long been recognized as one way of generating molecular diversity. But a recent paper (D. Schmucker et al., Cell 101, 1-20, 2000) sets what is surely a new record, with the identification of a Drosophila axon guidance receptor, termed Dscam, that has no fewer than 38,000 different isoforms. The extracellular domain is assembled in modular fashion, with multiple alternatives for each module (see diagram). Although it is possible that not all combinations are expressed in vivo, many clearly are: among 50 randomly selected Dscam cDNAs, 49 were unique.

The authors confirm that Dscam is involved in axon guidance, but whether the isoforms have different functions remains an open question. It is tempting to think that this molecular diversity is somehow related to the complexity of the neural wiring, but

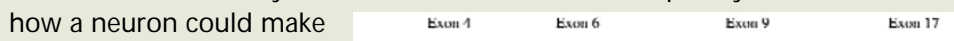
use of so many different possibilities-given the difficulties of specifying the splicing pattern with any precision-is still anyone's guess.

Charles Jennings

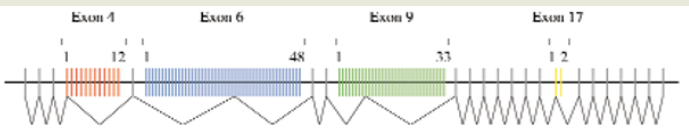

पमा

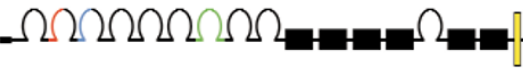

